\title{
Primary $\mathrm{PCl}$ during off-hours is not related to increased mortality
}

I(I) 33-39

(C) The European Society of Cardiology 2012

Reprints and permission:

sagepub.co.uk/journalsPermissions.nav

DOI: |0.1 |77/20488726/244|58|

acc.sagepub.com

QSAGE

\author{
Sanneke PM de Boer, Rohit M Oemrawsingh, \\ Mattie J Lenzen, Nicolas M van Mieghem, Carl Schultz, K Martijn \\ Akkerhuis, Maarten AH van Leeuwen, Felix Zijlstra, Ron $T$ van \\ Domburg, Patrick WJC Serruys and Eric Boersma (on behalf of \\ the interventional cardiologists of the Thoraxcenter 2000-2009)
}

\begin{abstract}
Aim: Previous studies have shown contradictory outcomes in ST-segment elevation myocardial infarction (STEMI) patients who underwent primary percutaneous coronary intervention ( $\mathrm{PPCl}$ ) during off-hours versus regular 'office' hours. We aimed to evaluate the relationship between $\mathrm{PPCl}$ timing (off-hours versus regular hours) and mortality in patients with STEMI undergoing PPCl.

Methods: The study population comprised 4352 consecutive STEMI patients treated with pPCl in a high-volume centre with a 24/7 programme during 2000-2009. Descriptive statistics and multivariable survival analyses were applied to evaluate the relationship between treatment during off-hours (Monday-Friday, 6.00 pm-8.00 am and weekends) versus regular hours and the incidence of all-cause mortality at 30-day and 4-year follow-up.

Results: A total of 2760 patients (63.4\%) were treated during off-hours and 1592 patients (36.6\%) during regular hours. With the exception of smoking, diabetes mellitus, use of glycoprotein Ilb/Illa antagonists and calcium antagonists, no major differences in baseline characteristics were observed between the groups. Mortality at 30-day follow-up was similar in patients treated during off-hours and those treated during regular hours $(7.7 \%$ vs $7.7 \%$; hazard ratio adjusted for potential confounders $1.03 ; 95 \% \mathrm{Cl} 0.82-1.28)$. Four-year mortality was similar (17.3\% vs $17.3 \%$; adjusted hazard ratio $0.95 ; 95 \% \mathrm{Cl} 0.8 \mathrm{I}-\mathrm{I} . \mathrm{II})$.
\end{abstract}

Conclusion: In STEMI patients who present during off-hours in a high-volume centre with $24 / 7$ service, $\mathrm{PPCl}$ provides similar survival as patients who were treated during regular hours.

\section{Keywords}

STEMI, off hours, primary percutaneous coronary intervention, outcome

Received: 10 January 2012; accepted 30 January 2012

\section{Introduction}

Randomised clinical trials have convincingly demonstrated that patients with ST-segment elevation myocardial infarction (STEMI) who undergo primary percutaneous coronary intervention (pPCI) have better event-free survival and clinical outcomes than those treated with fibrinolysis. ${ }^{1}$ In order to fully benefit from the instantaneous and long-term effects of pPCI, patients need to be treated as soon as possible after symptom onset. Guidelines recommend that pPCI be performed within 90 minutes of the first medical contact. $^{2-3}$ This recommendation is supported by several studies that reported a direct relation between (increased) time delay to pPCI and (worse) clinical outcome. ${ }^{4-6}$ Short onset-to-treatment times are best guaranteed in hospitals with an established interventional cardiology programme that offers full service 24 hours per day, 7 days per week $(24 / 7$ programme $) .^{2-3}$

Thoraxcenter, Department of Cardiology, Erasmus MC, Rotterdam, The Netherlands

\section{Corresponding author:}

Eric Boersma, Department of Cardiology, Thoraxcenter, Erasmus MC, 's Gravendijkwal 230, Room Bd38I, 3015 CE Rotterdam, The Netherlands.

Email: h.boersma@erasmusmc.nl 
Since the occurrence of STEMIs are more or less randomly distributed over time, in a $24 / 7$ programme, most patients will be treated during 'off-hours' - evening and night shifts and weekends. Previous studies have shown contradictory outcomes in STEMI patients who underwent pPCI during off-hours versus regular 'office' hours. However, most of these studies were conducted in centres using both fibrinolysis and pPCI for treatment of patients with STEMI, and did not evaluate long-term outcomes. ${ }^{7-18}$ In the year 2000, pPCI became the standard treatment for STEMI in our institution and a 24/7 programme was established. Baseline, procedural and follow-up data of all patients undergoing PCI in our institution were systematically collected. Consequently, we were able to evaluate the relationship between pPCI timing (off-hours versus regular hours) and short- and long-term outcome in STEMI patients.

\section{Methods}

\section{Patient population}

The Erasmus MC is a tertiary referral and teaching hospital in the broader region of Rotterdam (approximately 1.9 million inhabitants), located on the North bank of the Maas River. Between January 2000 and June 2004, the Erasmus $\mathrm{MC}$ was the only hospital in the region with pPCI facilities. From July 2004 onwards, the Maasstad hospital (also located in Rotterdam, on the south bank of the Maas River) started a 24/7 programme locally and provided regional pPCI service on Mondays and Thursdays and the first weekend of every month to improve service. The Erasmus $\mathrm{MC}$ provided pPCI service on the remaining days.

All consecutive patients 18 years or older who presented within 12 hours of symptom onset with ST-segment elevation myocardial infarction (STEMI) and who subsequently underwent pPCI in our institution between January 2000 and December 2009 were included in the analysis. STEMI is defined as patients presenting with ischaemic symptoms and persistent $(>20 \mathrm{~min})$ ST-segment elevation in at least two contiguous precordial leads or at least two adjacent limb leads by ECG. ${ }^{3}$ In total, 4541 pPCIs in 4352 patients were performed. In patients who were admitted more than once for pPCI $(n=189)$, only the initial procedure was used for this analysis.

\section{Patient management}

Patient management was in accordance with the applicable guidelines of the European Society of Cardiology (ESC). Patients received an aspirin and a loading dose of clopidogrel (300-600 mg) before pPCI and preferably in the ambulance. Clopidogrel ( $75 \mathrm{mg} /$ day) was given for at least one month in patients treated with bare metal stents (BMS), at least 3 months for patients treated with sirolimus-eluting stents (SES) and at least 6 months in patients treated with paclitaxel-eluting stents (PES) or everolimus-eluting stents
(EES). After the procedure, all patients were advised to remain on aspirin (>80 mg/day) indefinitely. Periprocedural glycoprotein IIb/IIIa antagonists were left to the discretion of the treating interventional cardiologist.

Since 2000, the interventional cardiology department has the policy of using one particular stent as default in a given time interval. The default stent between January 2000 and April 2002 was a BMS, between April 2002 and March 2003 a SES, between March 2003 and March 2007 a PES, and an EES since March 2007. Of note, during the study period a small number of STEMI patients was treated with another stent due to participation in a clinical trial comparing stents.

\section{Data collection}

According to the approved standard data-management procedures in our department, data are collected on demographics, cardiovascular history, clinical risk factors and treatment characteristics for all patients undergoing PCI and are stored in an electronic database. Data elements are filled out immediately after the completion of the PCI by the interventional cardiologist and the technician who assisted during the procedure. The database, which is maintained by a dedicated IT officer, is mainly designed for administrative purposes. A systematic evaluation of data completion and data integrity is implemented for data that are used for research purposes.

\section{Data management and follow-up}

Mortality data related to the entire cohort was obtained from interrogation of municipal civil registries between April and September 2011. A health questionnaire was subsequently sent to all living patients with specific inquiries on rehospitalisation and major adverse cardiovascular events (MACE). For patients who had adverse events at other centres, medical records or discharge summaries from the other institutions were systematically reviewed. General practitioners, referring cardiologists and patients were contacted in case further information was required.

\section{Endpoint definitions}

The primary endpoints were early mortality, which was defined as all-cause mortality within 30 days of the index event, and late mortality, which includes all-cause mortality at 1-year and 4-year follow-up. The secondary endpoints included repeat $\mathrm{PCI}$ (rePCI), coronary artery bypass grafting (CABG) or recurrent $\mathrm{MI}$ (reMI) and the composite endpoint of reMI, revascularisation (rePCI or CABG) and all-cause mortality at 30-day, 1-year and 4-year follow-up. ReMI at follow-up was diagnosed by recurrent typical clinical symptoms, the development of ST-segment elevation or left bundle branch block on electrocardiography with a CK-MB rise of three times the upper limit of normal and/or positive 
Table I. Baseline and procedural characteristics according to $\mathrm{PPCl}$ timing.

\begin{tabular}{|c|c|c|c|}
\hline & $\begin{array}{l}\text { Off-hours } \\
n=2760\end{array}$ & $\begin{array}{l}\text { Regular hours } \\
N=1592\end{array}$ & $p$-value \\
\hline Age (years $\pm S D$ ) & $60.9 \pm 12.8$ & $61.5 \pm 12.5$ & 0.14 \\
\hline Male $(\%, n)$ & $73.5,2029$ & $75.2,1197$ & 0.23 \\
\hline \multicolumn{4}{|l|}{ Medical history } \\
\hline Hypertension $(\%, n)$ & $40.1,1107$ & $38.6,614$ & 0.32 \\
\hline Hypercholesterolaemia & $72.4,1998$ & $71.4,1137$ & 0.49 \\
\hline Diabetes mellitus $(\%, n)$ & $13.0,358$ & $10.4,165$ & 0.011 \\
\hline Family history $(\%, n)$ & $28.6,790$ & $27.6,439$ & 0.46 \\
\hline Current smokers $(\%, n)$ & $41.8,1154$ & $36.0,573$ & $<0.001$ \\
\hline Previous MI $(\%, n)$ & I2.1, 334 & |3.1, 209 & 0.32 \\
\hline Previous $\mathrm{PCl}(\%, n)$ & $9.2,147$ & $7.9,217$ & 0.12 \\
\hline Previous CABG $(\%, n)$ & $2.4,66$ & $3.2,51$ & 0.11 \\
\hline Renal impairment $(\%, n)$ & $2.1,57$ & $1.8,29$ & 0.58 \\
\hline \multicolumn{4}{|l|}{ Procedural characteristics } \\
\hline Cardiogenic shock $(\%, n)$ & $4.9,135$ & $4.8,76$ & 0.86 \\
\hline Vessel disease & & & 0.63 \\
\hline I-vessel disease $(\%, n)$ & $54.8,1513$ & $55.2,878$ & \\
\hline 2-vessel disease $(\%, n)$ & $26.9,742$ & $27.6,440$ & \\
\hline 3 -vessel disease $(\%, n)$ & $18.3,505$ & I7.2, 274 & \\
\hline Multi-vessel disease $(\%, n)$ & $45.2,1247$ & $44.8,7 \mid 4$ & 0.83 \\
\hline Treated vessel & & & 0.35 \\
\hline Left main $(\%, n)$ & $3.5,97$ & $4.5,71$ & \\
\hline $\operatorname{RCA}(\%, n)$ & $34.0,938$ & $35.3,562$ & \\
\hline $\operatorname{LCX}(\%, n)$ & $14.3,396$ & $13.4,213$ & \\
\hline $\operatorname{LAD}(\%, n)$ & $46.6,1285$ & $44.9,715$ & \\
\hline Graft $(\%, n)$ & $0.6,17$ & $0.9,15$ & \\
\hline Glycoprotein Ilb/Illa antagonists $(\%, n)$ & $16.5,456$ & $21.4,340$ & $<0.001$ \\
\hline \multicolumn{4}{|l|}{ Discharge medication } \\
\hline Aspirin $(\%, n)$ & $90.1,2599$ & $90.2,1338$ & 0.91 \\
\hline Calcium antagonist $(\%, n)$ & 14.5378 & 19.8, 294 & $<0.001$ \\
\hline Beta-blockers $(\%, n)$ & $51.6,1342$ & $54.0,801$ & 0.14 \\
\hline RAAS-inhibitors $(\%, n)$ & $41.7,1085$ & $39.9,592$ & 0.25 \\
\hline Statins $(\%, n)$ & $71,6,1861$ & $71.2,1056$ & 0.79 \\
\hline
\end{tabular}

troponin levels in laboratory values. rePCI was defined as a repeat percutaneous intervention of any lesion located in the epicardial vessels. CABG was defined as a surgical intervention of any lesion located in the epicardial vessels.

\section{Statistical methods}

Off-hours were defined as weeknights (Monday to Friday from $6.00 \mathrm{pm}$ to $8.00 \mathrm{am}$ ) and weekends (from Friday 6.00 pm to Monday $8.00 \mathrm{am})$.

Continuous variables are presented as mean \pm standard deviation and categorical variables are expressed as numbers and percentages. Student's t-tests, Chi-square tests and Fisher's exact tests were applied to evaluate differences in baseline variables between patients treated during off-hours and regular hours, as appropriate.

We intended to obtain complete information in all patients, but failed to do so for the medication at discharge.
Using missing value analysis (MVA) we evaluated the extent of missing data and searched for patterns of missing data. MVA showed that there were $6.2 \%$ missing values on average for medication at discharge. For the patients with at least one of the variables of interest missing, we decided to impute the missing values by multiple imputation. ${ }^{19}$

The incidence of events over time was studied with the use of the Kaplan-Meier method, whereas log-rank tests were applied to evaluate differences between the treatment groups (treatment during off-hours versus regular hours). Patients lost to follow-up were considered at risk until the date of last contact at which point they were censored.

Cox proportional hazard $(\mathrm{PH})$ regression models were applied to evaluate the relationship between treatment during off-hours versus regular hours and the incidence of all-cause death at 30 days, 1 year and 4 years. The baseline clinical and procedural characteristics that are listed in Table 1 were considered as potential confounders for the 1-year and 4-year 

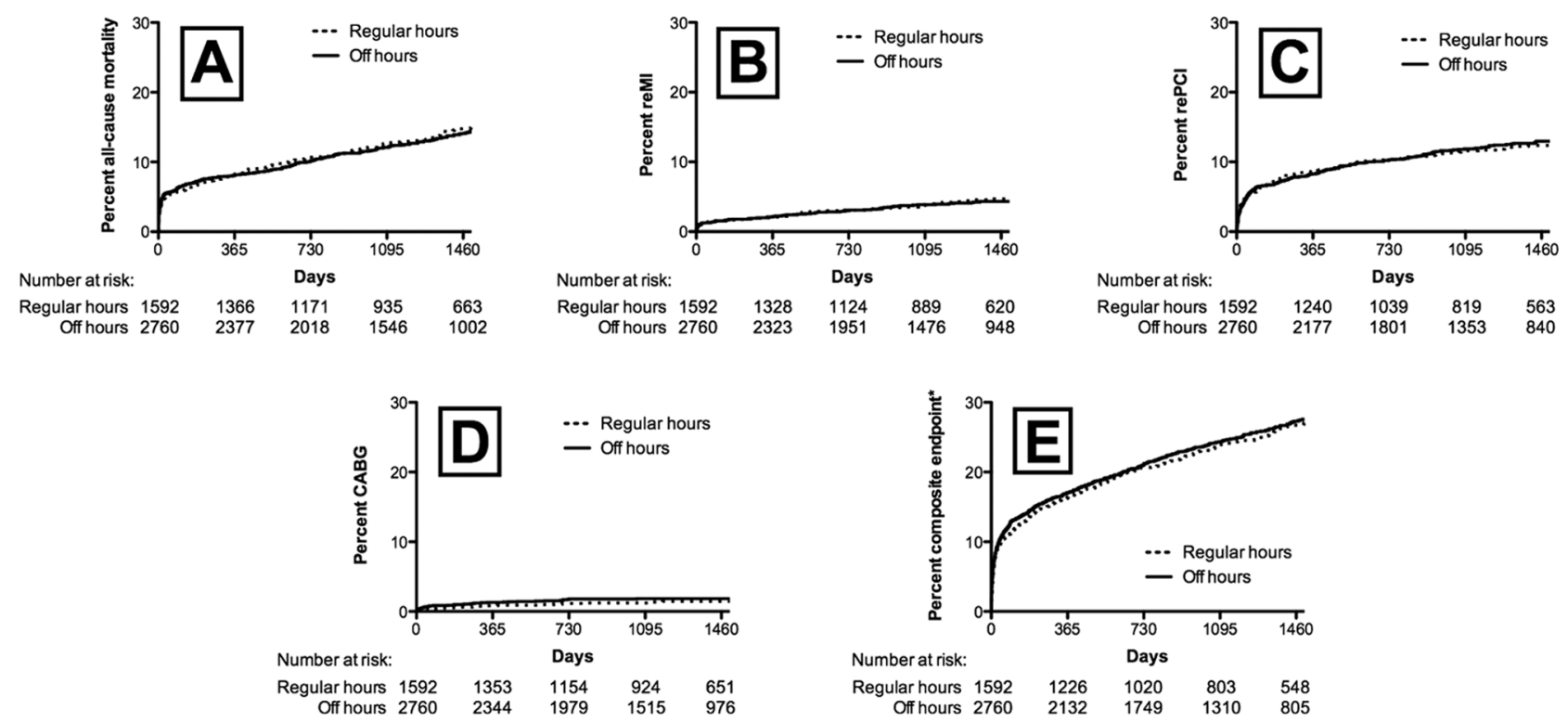

Figure I. Clinical outomes according to regular vs off hours. (A) all-cause mortality, (B) reMI, (C) rePCI, (D) CABG, (E) composite endpoint of all-cause mortality, reMI, rePCl and CABG.

mortality analysis. As the number of events was limited at 30 days, we were only able to adjust for the following clinically relevant factors: age, sex, multi-vessel disease, shock, previous MI, renal impairment and diabetes mellitus.

Final results are presented as adjusted hazard ratios (aHR) with 95\% confidence interval (CI). All statistical tests were two-tailed and a $p$-value $<0.05$ was considered significant. Statistical analyses were performed with SPSS for Windows version 17.0 (SPSS Inc, Chicago, Illinois, USA).

\section{Results}

\section{Key characteristics}

Between 1January 2000 and 31 December 2009, a total of 4352 consecutive patients presenting with STEMI underwent pPCI in our institution. A total of 2760 patients (63.4\%) were treated during off-hours and 1592 patients $(36.6 \%)$ during regular hours. Key characteristics of the two cohorts are presented in Table 1. With the exception of diabetes mellitus $(13.0 \%$ vs $10.4 \%, p=0.011)$, current smoking $(41.8 \%$ vs $36.0 \%, p<0.001)$ and use of glycoprotein IIb/IIIa antagonists $(16.5 \%$ vs $21.4 \%, p<0.001)$, no statistically significant differences in baseline and procedural characteristics were observed between the groups. It is noteworthythat the percentage of patients presenting with cardiogenic shock was similar in both groups $(4.9 \%$ and $4.8 \%$, respectively, $p=0.86$ ). There were also no statistically significant differences in discharge medication, except for the use of calcium antagonists $(14.5 \%$ vs $19.8 \%, p<0.001)$.

\section{Mortality}

Information on survival status at 1-year follow-up was complete for $96.5 \%$ of patients. The median follow-up period was 1246 days (IQR 651-2228 days).

The cumulative incidence of all-cause mortality at 30-day follow-up was similar in the patients treated during off-hours and those treated during regular hours $7.7 \%$ vs $7.7 \%$ respectively (Kaplan Meier estimates). Similarly, no statistically significant differences were observed in allcause mortality at 1 -year (10.9\% vs $12.5 \%)$ and 4-year follow-up (17.3\% vs $17.3 \%)$. In fact, the cumulative incidence curves were superimposed throughout the entire 4-year follow-up period (Figure 1A). Multivariable adjustment for potential confounders of the relation between treatment timing and the incidence of all-cause mortality did not change this finding (aHR at 4 years 0.96 and $95 \%$ CI $0.81-1.12$ ).

\section{Non-fatal and composite endpoints}

We did not find any clinically relevant difference in the incidence of non-fatal endpoints between patients treated during off-hours vs regular hours. Results were similar at short-term and long-term follow-up (Table 2, Figure 1BD). At 4-year follow-up, the crude cumulative incidences of reMI were $5.5 \%$ and $4.6 \%$, the incidences of CABG were $1.6 \%$ and $3.0 \%$, and the incidences of rePCI were $12.9 \%$ and $13.4 \%$, respectively. Again, cumulative incidence curves were superimposed for all these endpoints. Treatment timing had no contribution in multivariable Cox PH models that related patient characteristics with nonfatal clinical outcomes. Adjusted HRs of treatment timing 


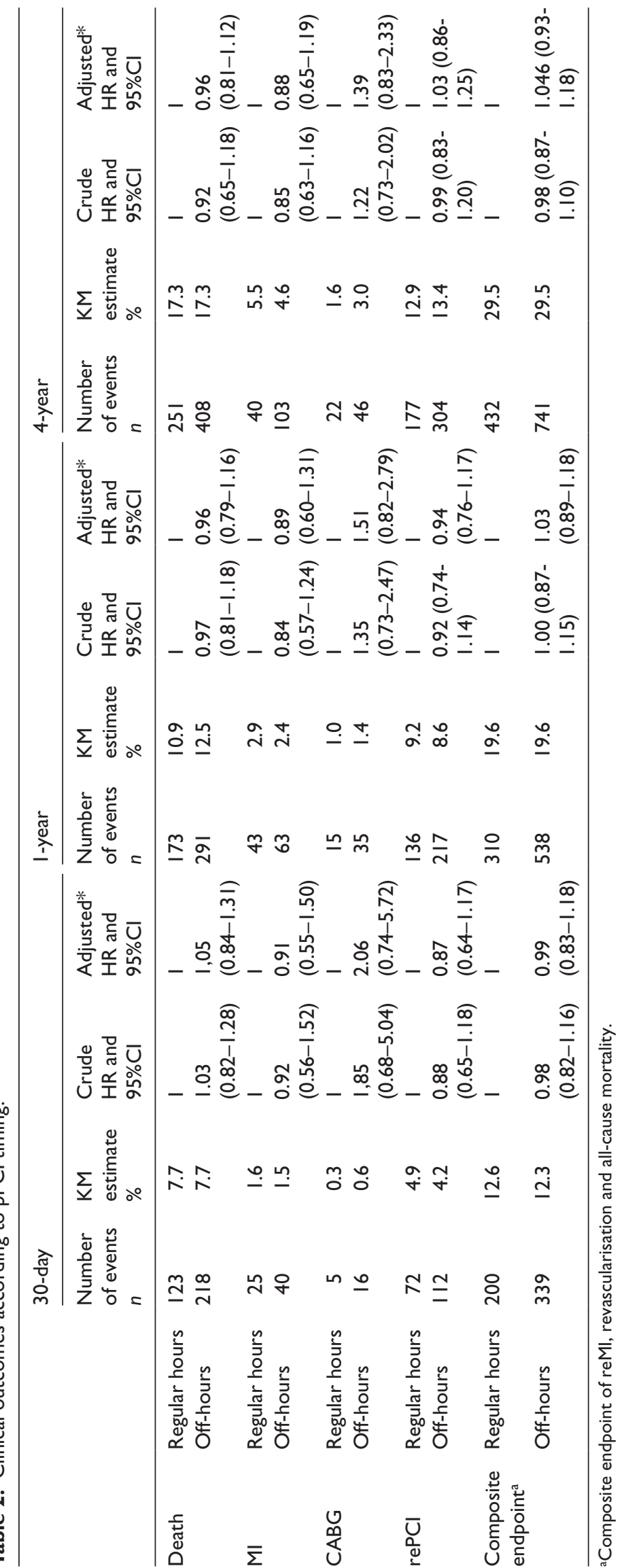

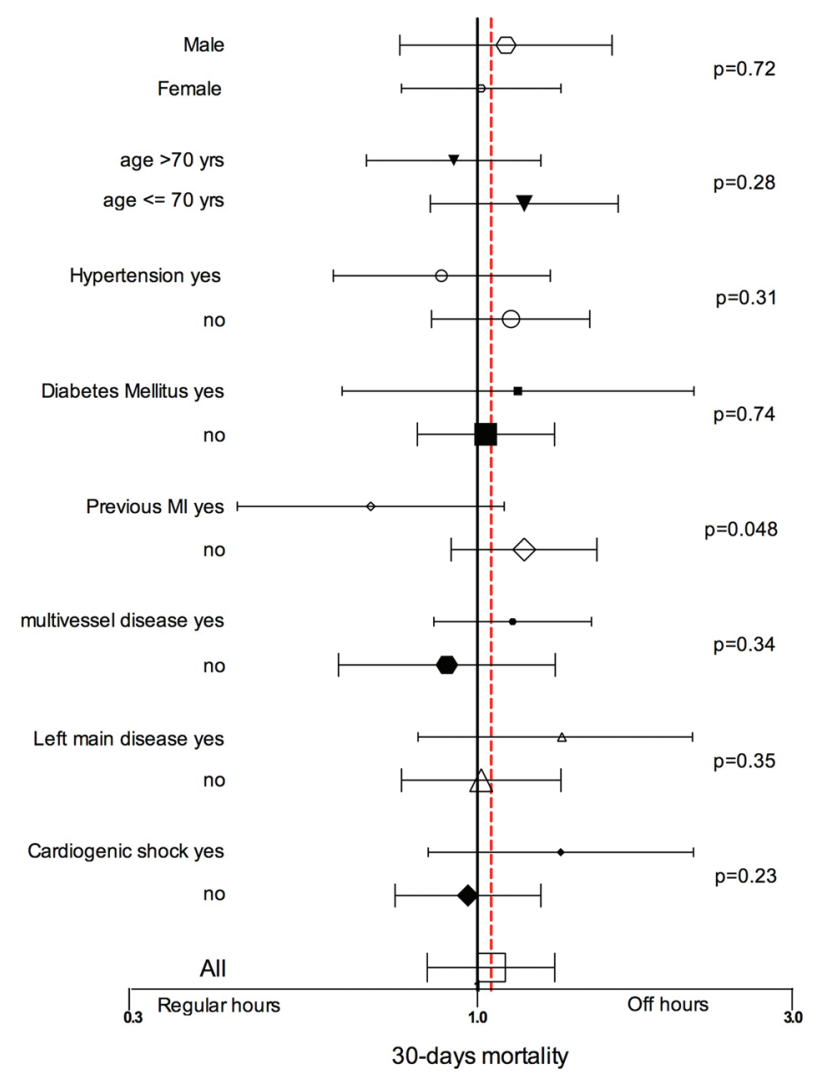

Figure 2. Treatment during off hours in different subgroups.

were not significant and close to 1 for all endpoints at all three follow-up times that we studied.

The cumulative incidence of the composite endpoint of all-cause death, reMI, PCI or CABG at 4-year follow-up was $29.5 \%$ in patients treated during off-hours and $29.5 \%$ in those treated during regular hours (Table 2, Figure 1E). The aHR was 1.05 and the $95 \%$ CI ranged from 0.93 to 1.18 , indicating that there was no association between treatment timing and the incidence of this composite endpoint.

\section{Findings in subgroups}

Figure 2 shows the relationship between treatment timing and 30-day all-cause mortality in a number of clinically relevant subgroups. All in all, in the subgroups that we considered, we found no major deviations from the overall result. Except in the category relating to the history of MI, $95 \%$ confidence intervals of treatment effect were largely overlapping, and none of the formal heterogeneity tests was statistically significant.

\section{Discussion}

In this long-term follow-up study of STEMI patients who were treated during 2000-2009 in a high-volume centre with 
24/7 service, clinical outcomes were similar in patients undergoing pPCI during off-hours and during regular hours. This similarity in outcome was already observed at 30 days and was maintained until 4 years after the initial procedure. Consistent results were seen in clinically relevant subgroups, including the elderly and patients with multi-vessel disease.

The quality of care delivered to STEMI patients may differ during day and night because of variations in door-toballoon time, performance of physicians, catheterisation laboratory and coronary care staff. Compared to regular office hours, hospital staffing is generally reduced during nights and on weekends compared with weekdays. The short-term outcome (after adjustment for any differences in case mix) may be regarded as a proxy measure of the quality of care during the procedure, whereas the long-term outcome to a greater extent depends on the development of the disease, the use of long-term medication and (probably) the use of coronary revascularisations. Interestingly, we found few differences in baseline characteristics between patients treated during off-hours and regular hours. Also medical treatment at discharge was similar. Apparently, in the region of Rotterdam, STEMI patients constitute a homogeneous population, regardless of the timing of presentation. Consequently, the point estimates of the effect of treatment timing did not change after adjustment for patient characteristics. The estimates of the effect for all the endpoints indicated that treatment during off-hours is as safe and effective as treatment during regular hours.

Previous studies have shown contradictory results in outcome in STEMI patients who underwent pPCI during offhours versus regular hours. Whereas some studies showed that presentation and treatment during off-hours only had limited impact on in-hospital mortality, $7,9,12,13,15,16,18$ other studies revealed higher in-hospital mortality in pPCI patients during off-hours than regular hours. ${ }^{8,10,11,14} \mathrm{~A}$ straightforward comparison of these clinical studies is complicated by differences in patient characteristics and (medical) co-treatment, as well as by the applied definitions. For example, Henriques et al, who reported higher mortality after off-hour treatment, focused on the circadian patterns of symptom onset, including routine duty hours $(0800$ 1800) versus off-hours irrespective of day of the week. ${ }^{8}$ In the study by Kostis et al, ${ }^{10}$ the significant difference in 30-day mortality for patients treated during off-hours disappeared after adjustment for the use of invasive cardiac procedures, which appeared less often in patients admitted at weekends. Furthermore, in some of the previous studies, patients were not only treated with pPCI, but also with fibrinolysis, which may have led to different results. ${ }^{9-11,15,18}$ Since 2000 in our centre, pPCI is the default strategy for all STEMI patients on a $24 / 7$ basis, precluding potential bias due to fibrinolysis. On the other hand, most patients treated in our centre are referred for pPCI by hospitals in the larger region of Rotterdam that do not provide a $24 / 7$ pPCI service. Although, apparently, the referral pattern is not dependent on the day of the week and the time of the day (as we found no differences in patient characteristics in relation to treatment timing), the patient selection by referral hospitals might partly explain the consistent outcomes.

\section{Study limitations}

Other reported predictors of outcome after pPCI include time delay from symptom onset to the first balloon inflation $^{4-6}$ and physician volume..$^{20,21}$ Since the database that we used for our study was not specifically designed to address these issues, several relevant quality parameters have not been recorded prospectively. Still, we retrospectively recorded the time between hospital admission and the start of the pPCI procedure (data were available for $70 \%$ of patients), and we found no differences between patients treated during off-hours versus those treated during regular hours (data not shown). The presented results are based on a single-centre experience, which limits the external validity. Nevertheless, the Thoraxcenter Rotterdam can be considered representative for larger tertiary referring and teaching (academic) hospitals in Western populations.

For the follow-up on non-fatal endpoints we were dependent on the responses of patients on health questionnaires that were systematically sent to all living patients, with specific inquiries on rehospitalisation and MACE. Thus, we might have missed some non-fatal endpoints, particularly those that did not result in hospital admissions. We have no indication that under-reporting (if any) was related to the timing of the initial treatment. This phenomenon might have resulted in effect estimates that are biased towards the null. Still, we are confident that similar effects were seen for all ('hard' and 'softer') endpoints.

\section{Conclusion}

STEMI patients who were treated in a high-volume centre with a 24/7 programme have similar short- and long-term outcomes whether they are treated during off-hours or regular hours. Our findings, which are based on systematic monitoring of treatment outcome results, do not necessitate us changing our practice. Instead, these results may encourage other centres to expand their service.

\section{Acknowledgements}

The authors thank the senior cardiologists who performed the procedures during the study period: Eugene McFadden, Pim J de Feyter, Peter PT de Jaegere, Robert Jan van Geuns, Evelyn Regar, Georgios Sianos, Pieter C Smits, Martin J van der Ent, Willem J van der Giessen, Carlos A van Mieghem, Henricus J Duckers, Atilla Dirkali, Arie G de Vries, and the cardiac catheterisation staff of the Thoraxcenter.

\section{Funding}

This research received no specific grant from any funding agency in the public, commercial or not-for-profit sectors. 


\section{Conflict of interest}

None declared by authors.

\section{References}

1. Keeley EC, Boura JA and Grines CL. Primary angioplasty versus intravenous thrombolytic therapy for acute myocardial infarction: a quantitative review of 23 randomised trials. Lancet 2003; 361:13-20.

2. Smith SC, Jr, Feldman TE, Hirshfeld JW, Jr, et al, American College of Cardiology/American Heart Association Task Force on Practice G, Intervention AASWCtUtGfPC. ACC/ AHA/SCAI 2005 guideline update for percutaneous coronary intervention: a report of the American College of Cardiology/American Heart Association Task Force on Practice Guidelines (ACC/AHA/SCAI Writing Committee to Update the 2001 Guidelines for Percutaneous Coronary Intervention). J Am Coll Cardiol 2006; 47: e1-121.

3. Van de Werf F, Bax J, Betriu A, et al, Guidelines ESCCfP. Management of acute myocardial infarction in patients presenting with persistent ST-segment elevation: the Task Force on the Management of ST-Segment Elevation Acute Myocardial Infarction of the European Society of Cardiology. Eur Heart J 2008; 29: 2909-45.

4. Cannon CP, Gibson CM, Lambrew CT, et al. Relationship of symptom-onset-to-balloon time and door-to-balloon time with mortality in patients undergoing angioplasty for acute myocardial infarction. JAMA 2000; 283: 2941-47.

5. De Luca G, Suryapranata H, Ottervanger JP, et al. Time delay to treatment and mortality in primary angioplasty for acute myocardial infarction: every minute of delay counts. Circulation 2004; 109: 1223-25.

6. De Luca G, Suryapranata H, Zijlstra F, et al, Group ZMIS. Symptom-onset-to-balloon time and mortality in patients with acute myocardial infarction treated by primary angioplasty. J Am Coll Cardiol 2003; 42: 991-97.

7. Berger A, Meier JM, Wasserfallen JB, et al. Out of hours percutaneous coronary interventions in acute coronary syndromes: long-term outcome. Heart 2006; 92: 1157-58.

8. Henriques JP, Haasdijk AP and Zijlstra F, Zwolle Myocardial Infarction Study G. Outcome of primary angioplasty for acute myocardial infarction during routine duty hours versus during off-hours. J Am Coll Cardiol 2003; 41: 2138-42.

9. Jneid $\mathrm{H}$, Fonarow $\mathrm{GC}$, Cannon $\mathrm{CP}$, et al, Get With the Guidelines Steering $C$, Investigators. Impact of time of presentation on the care and outcomes of acute myocardial infarction. Circulation 2008; 117: 2502-9.

10. Kostis WJ, Demissie K, Marcella SW, et al, Myocardial Infarction Data Acquisition System Study G. Weekend versus weekday admission and mortality from myocardial infarction. N Engl J Med 2007; 356: 1099-1109.
11. Magid DJ, Wang Y, Herrin J, et al. Relationship between time of day, day of week, timeliness of reperfusion, and inhospital mortality for patients with acute ST-segment elevation myocardial infarction. JAMA 2005; 294: 803-12.

12. Ortolani P, Marzocchi A, Marrozzini C, et al. Clinical comparison of "normal-hours" vs "off-hours" percutaneous coronary interventions for ST-elevation myocardial infarction. Am Heart J 2007; 154: 366-72.

13. Sadeghi HM, Grines CL, Chandra HR, et al. Magnitude and impact of treatment delays on weeknights and weekends in patients undergoing primary angioplasty for acute myocardial infarction (the cadillac trial). Am J Cardiol 2004; 94: 637-40, A639.

14. Saleem MA, Kannam H, Aronow WS, et al. The effects of off-normal hours, age, and gender for coronary angioplasty on hospital mortality in patients undergoing coronary angioplasty for acute myocardial infarction. Am J Cardiol 2004; 93: 763-64.

15. Zahn R, Schiele R, Seidl K, et al. Daytime and nighttime differences in patterns of performance of primary angioplasty in the treatment of patients with acute myocardial infarction. Maximal Individual Therapy in Acute Myocardial Infarction (MITRA) Study Group. Am Heart $J$ 1999; 138: 1111-17.

16. Casella G, Ottani F, Ortolani P, et al. Off-hour primary percutaneous coronary angioplasty does not affect outcome of patients with ST-Segment elevation acute myocardial infarction treated within a regional network for reperfusion: The REAL (Registro Regionale Angioplastiche dell'Emilia-Romagna) registry. JACC Cardiovasc Interv 2011; 4: 270-78.

17. Glaser R, Naidu SS, Selzer F, et al. Factors associated with poorer prognosis for patients undergoing primary percutaneous coronary intervention during off-hours: biology or systems failure? JACC Cardiovasc Interv 2008; 1: 681-88.

18. Bell CM and Redelmeier DA. Mortality among patients admitted to hospitals on weekends as compared with weekdays. N Engl J Med 2001; 345: 663-68.

19. Sterne JA, White IR, Carlin JB, et al. Multiple imputation for missing data in epidemiological and clinical research: potential and pitfalls. BMJ 2009; 338: b2393.

20. Magid DJ, Calonge BN, Rumsfeld JS, et al, National Registry of Myocardial I, Investigators. Relation between hospital primary angioplasty volume and mortality for patients with acute MI treated with primary angioplasty vs thrombolytic therapy. JAMA 2000; 284: 3131-38.

21. Canto JG, Every NR, Magid DJ, et al. The volume of primary angioplasty procedures and survival after acute myocardial infarction. National Registry of Myocardial Infarction 2 Investigators. $N$ Engl J Med 2000; 342: 1573-80. 